\title{
Gene-environment interactions in other-race face recognition: Early caregiving experience and oxytocin receptor genotype interact to increase speed of other-race categorization
}

\author{
Michelle Jin-Yee Neoh ${ }^{1,+}$, Peipei Setoh ${ }^{1,+, *}$, Andrea Bizzego ${ }^{2}$, Moses Tandiono ${ }^{3,4}$, Jia \\ Nee Foo ${ }^{3,4}$, and Gianluca Esposito ${ }^{1,2,3, * *}$ \\ ${ }^{1}$ Psychology Program, School of Social Sciences, Nanyang Technological University, Singapore, Singapore \\ ${ }^{2}$ Department of Psychology and Cognitive Science, University of Trento, Rovereto, Italy \\ ${ }^{3}$ Lee Kong Chian School of Medicine, Nanyang Technological University, Singapore, Singapore \\ ${ }^{4}$ Human Genetics, Genome Institute of Singapore, A*STAR, Singapore, Singapore \\ *psetoh@ntu.edu.sg \\ **:gianluca.esposito@ntu.edu.sg \\ +These authors contributed equally to this work
}

\begin{abstract}
The other-race face recognition deficit is a robust finding in the literature on facial processing in humans. Although previous models of the other-race effect have proposed the role of experience and interracial contact, genetics have not been examined in the context of other-race face recognition. The aim of this study was to investigate the gene-environment interaction between early caregiving experience and oxytocin receptor gene genotypes with other-race face recognition in adults. Eighty-nine Singaporean adults gave information on their early caregiving experiences with own- and other-race caregivers and genotyping of their oxytocin receptor gene (rs53576) was also conducted. Participants completed a visual categorization task where they identified the race of a face (Chinese or Javanese) and their categorization response time was measured. A significant main effect of early caregiving experience was found where reaction time was significantly slower in individuals with no other-race caregiving experience than individuals with other-race caregiving experience. In addition, only non-G carriers of rs53576 without other-race caregiving experience had a significantly slower reaction time compared to non-G carriers with other-race caregivers. This was not observed in G carriers, indicating a gene-environment interaction. These results highlight the role of early interracial contact on other-race face recognition and its interaction with genetics. Future studies can employ a longitudinal design for further insight into this gene-environment interaction across development.
\end{abstract}

\section{Introduction}

Faces have a homogenous positioning of internal elements regardless of the individual, and early in life, human infants are attracted to faces and face-like stimuli ${ }^{1,2}$. Human adults are highly proficient in the recognition and processing of face stimuli such that the categorization of faces as belonging to one's own social group or not is a rapid and automatic process $^{3}$. A robust finding that has been consistently demonstrated is an other-race recognition deficit, which has been termed as the other-race effect (ORE) ${ }^{4}$, which is a tendency for recognition accuracy to be higher for same-race faces than for cross-race faces. The ORE has real-world repercussions such as difficulties in social interaction, inaccurate eyewitness testimony $^{5}$ and implicit racism ${ }^{6,7}$. Two theoretical models have been proposed to explain the ORE; perceptual expertise models and social categorization models. Perceptual expertise models argue that differences in contact with same race versus other race faces lead to differential expertise in the processing and recognition accuracy of other-race faces ${ }^{4,8}$. On the other hand, social cognitive models argue that the ORE arises due to differences in the social cognitions involved in processing ingroup versus outgroup members ${ }^{9}$.

In line with perceptual expertise models, previous research suggests that early experience plays a critical role in shaping children's categorization of faces. An individual's ability to categorize faces based on social concepts such as race stems from early, repeated experiences and develops through socialization processes ${ }^{10-12}$. Firstly, most children have asymmetrical visual exposure to faces of different races where they see own-race faces at a disproportionately higher frequency than other-race faces. Studies have found that the ORE emerges early where from 9 months onwards, where infants not only classified own-race and other-race faces into separate categories ${ }^{13,14}$ but they also process own-race faces 
as individuals more compared to other-race faces. Secondly, significant improvement in other-race face recognition and reduction in the ORE with training in other-race face individuation has been documented in infants ${ }^{15,16}$. On the other hand, social cognitive models propose that the ORE arises due to differences in the type of social cognitive processing involved towards ingroup and outgroup members where ownrace (ingroup) faces tend to be processed at an individualistic level, and other-race (outgroup) faces tend to be processed at a more superficial, categorical level ${ }^{9}$. It has been suggested that weaker encoding of individuating features of other-race faces relative to own-race faces may occur as the result of categorizing other races as outgroups ${ }^{17}$. For example, studies consistently find that individuals pay attention to identitydiagnostic characteristics such as configural information in the eye region when encoding own-race faces ${ }^{18}$.

In an attempt to integrate these two theoretical models, the Categorization-Individuation model ${ }^{18}$ proposes that the ORE is a multiply-determined phenomenon, which is the product of a tendency to selectively attend to identity-diagnostic characteristics among same-race faces but category-diagnostic features among other-race faces. The model argues that three distinct, empirically supported factors: (i) social categorization, (ii) perceiver motivation and (iii) perceiver experience with discriminating same-race and other-race faces, work together in driving this selective attention during face encoding and consequently, affecting face recognition. The central premise is that during face encoding, there are two ways of processing faces - categorization and individuation. In the context of face processing, categorization involves classifying faces by race whereas individuation refers to discriminating individual faces within a racial group. The level of individuation experience - prior experience in discriminating among faces of a particular race - has been suggested to facilitate an individual's ability and efficiency in extracting identitydiagnostic information from faces. In a similar vein, the perceptual-social linkage hypothesis ${ }^{19}$ suggests that increased perceptual exposure to own-race faces combined with mostly positive experiences associated with these faces, leads to the development of implicit racial bias - favouring one's own race over others. This hypothesis suggests that levels and type of perceptual exposure has a role in influencing an individual's social cognitions. As such, since asymmetrical perceptual exposure and its associated experiences has a possible role in shaping social cognitions, this could possibly indicate that perceptual exposure can influence the ORE through shaping social cognitions.

Real-life experiences have been proposed to translate to superiority in other-race face recognition and also to play a critical role acquiring face expertise ${ }^{20}$ and recognition accuracy. However, the empirical support for the prediction that the size of the ORE will vary with amount of interracial contact experienced in an individual's everyday life appears to be contradictory. Studies have shown both significant ${ }^{21-23}$, and non-significant ${ }^{24,25}$ correlations of the ORE with interracial contact. Moreover, a meta-analysis calculated that other-race contact explained only $2 \%$ of variation in the size of the ORE $\mathrm{O}^{4}$. However, results from a recent study suggest that there is a critical period for other-race face recognition ${ }^{26}$. Their results demonstrated that after approximately 12 years of age, extended other-race social contact as an adult - even over several years and involving many other-race friends produced no improvement in other-race recognition and suggested that early childhood exposure was the most sensitive period for the development of other-race face recognition. Therefore, taking together these results and findings regarding individuation experience in other-race faces, there appears to be a gap in research on the influence of specific early childhood experiences on other-race face recognition. The present study aims to examine early caregiving experience on other-race face recognition accuracy in Singaporean adults.

In this study, early caregiving experience is defined as being cared for during infancy or childhood by a kin or non-kin caregiver other than one's own parents. Singapore provides a unique context for examining the impact of early childhood caregiving experience due to the demographic profile of caregivers in the country. Child caregiving arrangements involving caregivers other than parents are a common practice in Singapore due to a number of reasons. With higher levels of education among women ${ }^{27}$ and the trend of increasing full-time, female labor force participation ${ }^{28}$, mothers in Singapore have to balance both their work and caregiving responsibilities ${ }^{29,30}$. As a result, infants and/or children are often placed in the care of (i) grandparents, (ii) foreign domestic helpers and (iii) childcare centres, where children will have contact with other races in the cases of (ii) and (iii). Taken together, the prevalence of early other-race caregiving experience and a critical period during childhood for other-race face recognition highlight the importance of investigating the role of early caregiving experience on otherrace face recognition in Singapore. In line with perceptual expertise models and results indicating a critical period in childhood for other-race face recognition ${ }^{26}$, we expect that individuals who had an other-race caregiver will have more accurate and efficient other-race face recognition compared to those with same-race caregivers and those without caregiving experience. While the integrated model includes aspects of learning and social cognition, it does not include a genetics perspective in explaining findings on the ORE even though responses to social stimuli can be thought to be the product of both environmental and genetic factors. There is a significant body of research documenting the genetic factors that have been found to be associated with social cognitions, and certain genes have also been associated with the expression of social sensitivities to the environment. In line with tenets of the Categorisation-Individuation model regarding differences in the social cognition involved in processing 
same-race and other-race faces, it follows that genetic factors which have been associated with social cognitive processes could possibly influence the ORE. The oxytocin system has been implicated in human socially related personality traits and behaviors ${ }^{31,32}$. Specifically, the oxytocin receptor gene (OXTR) has been identified as a candidate gene regulating attachment-related behaviors and social cognitions in humans where the link between single nucleotide polymorphisms (SNPs) in this gene and sociality has been examined ${ }^{33,34}$ One such SNP is rs53576 (G/A), which previous studies have found $\mathrm{G}$ allele homozygotes (GG) to be associated with higher (i) self-reported empathy ${ }^{35}$, (ii) prosocial features ${ }^{36}$ and (iii) general sociality as rated by peers ${ }^{37}$ as compared to A allele carriers (AA/AG). While these findings point towards the influence of the OXTR genotype on social behaviors, the effect of this SNP genotype specifically on the ORE has not been studied previously. In addition, there have been some mixed findings about the association of face recognition and another OXTr SNP; rs237887 38,39 , which suggest a relationship between OXTr genotypes and face recognition. Hence, this study investigated the role of the rs53576 in the OXTR gene on the ORE and its possible interactions with early caregiving experience. We expect that non-G carriers of rs53576 with other-race caregivers will have more accurate other-race face recognition compared to those with same-race caregivers and those without caregiving experience.

The central premise of the Categorisation-Individuation model $^{18}$ is that during face encoding, there are two ways of processing faces - categorization and individuation. The model proposes multiple determinants of the ORE and integrates two main perspectives: (i) perceptual expertise and (ii) social cognitions. It also acknowledged the role of (i) perceiver characteristics, specifically experience with individuation of a particular race's face and (ii) social cognitions underlying social categorisation in influencing categorization and individuation processes. Although exposure to other-race faces and interracial contact were suggested to influence perceiver's individuation experience and social categorization, there remain other factors that could similarly influence a perceiver's experience and social cognitions. Hence, we proposed a number of additions to be made to this existing model. First, we propose the inclusion of a genetics perspective and possible gene-environment interactions in explaining the other-race face recognition through their influence on face processing, social information processing and social cognitions. With the understanding that responses to face stimuli can be thought of as a product of genetic and environment factors, the present study aims to investigate both genetic and environmental factors as well as their interactions on other-race face recognition. The present study examined the oxytocin system, which has been implicated in human socially related personality traits and behaviours. Specifically, the oxytocin receptor gene (OXTR) has been identified as a candidate gene regulating attachment-related behaviours and social cognitions in humans. Hence, the rs53576 SNP genotype was examined in the present study as a possible candidate gene in other-race face recognition. Second, the present study examined early caregiving experience as an environmental factor that involves interracial contact and exposure to other-race faces, which could possibly influence other-race face recognition. Lastly, the possible gene-environment interactions between early caregiving experience and rs53576 genotypes, which have not been previously studied, were examined. In summary, while the Categorisation-Individuation model accounts for and integrates perceptual expertise and social cognitive models, it does not take into consideration genetic factors that may influence perceptual expertise and social cognitions. Figure 1 illustrates the existing model along with key characteristics of the present study and how the present study aims to address this gap in the existing model.

The present study aims to examine the effect of genetic factors, environment factors and their interactions on the ORE in Singapore. Specifically, we investigated the effects of early caregiving experiences and the genotypes of OXTR in Singaporean adults in influencing adults' categorization of own-race and other-race faces (see Figure 1). Based on past findings supporting perceptual expertise models, we postulate that increased interracial contact and exposure to other-race faces will result in greater expertise and efficiency in distinguishing other-race faces. Greater exposure to other-race faces through increased interracial contact with one or more other-race caregivers allows individual to gain experience and expertise in distinguishing same-race and other-race faces, thus improving other-race face recognition. Since social categories can be accurately extracted even under suboptimal viewing conditions ${ }^{40}$, the accuracy of categorizing same versus other race faces will likely be equally high in participants given sufficient time. Hence, the time taken for visual categorization of the face stimuli by race was measured to determine the differences in the efficiency with which these category-diagnostic features are extracted by different individuals.

Firstly, we hypothesized that there will be significant differences in the reaction times for race categorization between individuals with different caregiving experiences. In Hypothesis 1 , we expect that individuals with other-race caregiving experience will exhibit faster race categorization when presented with face stimuli of different races compared to individuals with same-race caregivers or no caregiving experience. Secondly, from previous studies, the $\mathrm{G}$ allele of rs53576 appears to be associated with prosocial traits including higher sociality and social cognitive processing. Hence, we hypothesized that there will be significant differences in the reaction times for race categorization between non-G carriers of the OXTR rs53576 SNP with different early caregiving experiences. In Hypothesis 2, we expect that non-G carriers of rs53576 with an other-race caregiver will categorize faces of different races 


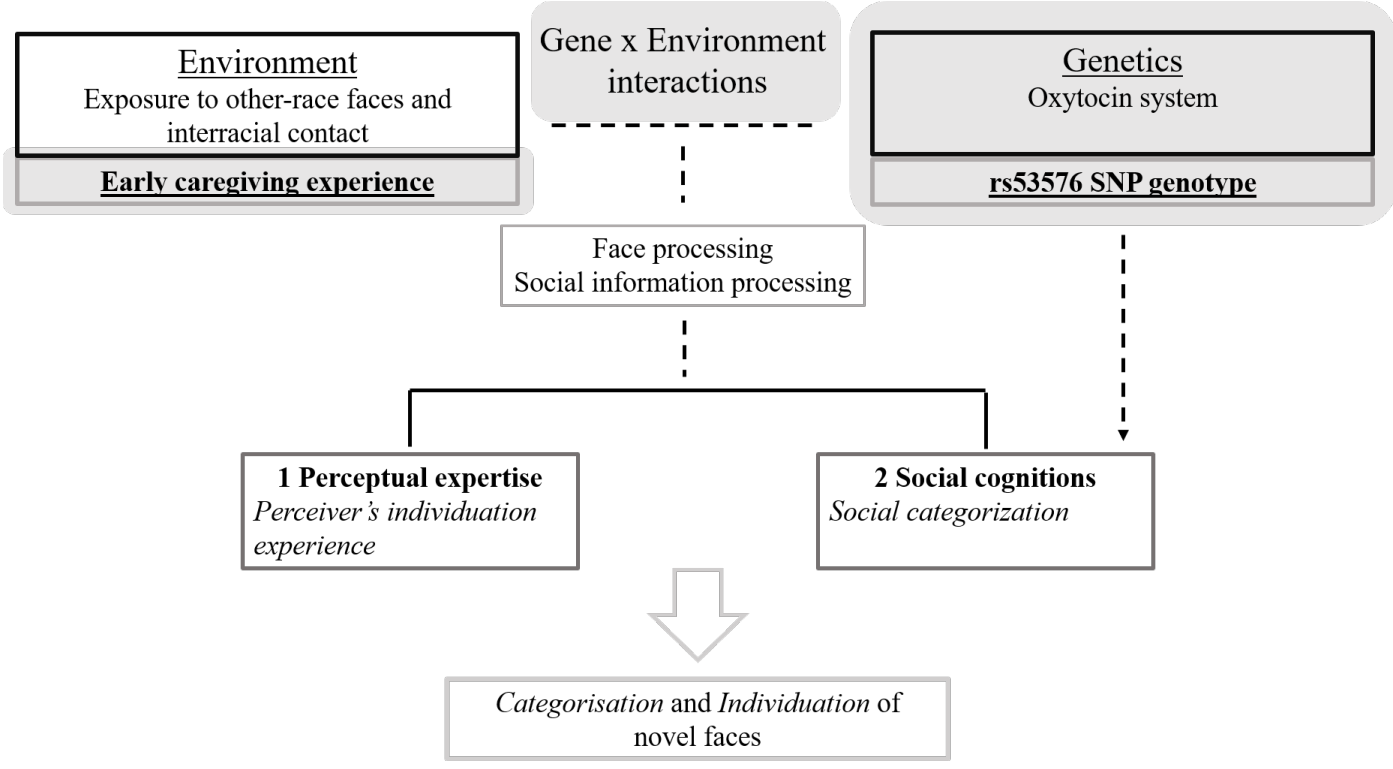

Figure 1. Diagram of the main features of the Categorisation-Individuation model (Hugenberg et al, 2014) with proposed additions to the model and key research variables of the present study highlighted in grey in the figure.

faster than non-G carriers with same-race or no caregiving experience.

\section{Results}

\section{Early caregiving experience}

A significant main effect of early caregiving experience on visual categorization response latency was found $(\mathrm{F}(2,83)$ $=6.30, \mathrm{p}=.003$ ) (Figure 2). Post-hoc Mann-Whitney tests were run to investigate the effect. Results from these post-hoc tests indicated that reaction time was significantly slower in individuals with no caregiving experience than individuals with an other-race caregiver $(\mathrm{U}=411, \mathrm{p}=.001)$. The other pairwise comparisons showed that there were no significant differences in reaction time between (i) individuals with no caregiving experience and those with a same-race caregiver ( $\mathrm{U}$ $=200, \mathrm{p}=.133$ ) and between (ii) individuals with same-race caregivers and those with an other-race caregiver $(\mathrm{U}=214, \mathrm{p}$ $=.119$ ).

\section{rs53576}

There was no significant main effect of the OXTR rs53576 genotype on $(\mathrm{F}(1,83)=0.42, \mathrm{p}=.52)($ Figure 3$)$. However, a significant interaction effect between OXTR rs53576 genotype and early caregiving experience was found $(\mathrm{F}(2,83)=$ $3.57, \mathrm{p}=.03)$. There was a significant difference between non-G carriers with other-race caregivers compared to non-G carriers with no caregiving experience. Non-G carriers with no caregiving experience had a significantly slower reaction time compared to non- $\mathrm{G}$ carriers with other-race caregivers ( $\mathrm{U}$ $=76, \mathrm{p}<.001$ ) (Figure 4). However, this difference between individuals with other-race caregivers and those without caregiving experience was not significant in $\mathrm{G}$ carriers $(\mathrm{U}=134$, $\mathrm{p}=.271$; Figure 4).

\section{Discussion}

The present study aimed to investigate the role of both genetic and environmental factors on other-race face recognition where we examined the rs53576 OXTr genotype and early caregiving experience in Singaporean adults. Here, we proposed the inclusion of a genetics perspective to be considered in addition to existing models and theories, in investigating other-race face recognition.

A significant main effect of early caregiving experience was found where there was a significant difference in otherrace face recognition between individuals with no caregiving experience compared to those with other-race caregivers. This finding supports Hypothesis 1 and suggests that greater early life other-race exposure and contact can improve otherrace face recognition. We propose that greater exposure to other-race faces through increased interracial contact with an other-race caregiver allows individual to gain experience and expertise in distinguishing same-race and other-race faces, thus improving other-race face recognition. This explanation also aligns with perceptual expertise models in that increased interracial contact, specifically early caregiving experience with other-race caregivers, can improve other-race face recognition and reduce the size of the ORE. Notably, findings from this study also dovetail with the results indicating a critical period in early childhood for other-race face recognition ${ }^{26}$ given that early caregiving experience measured in this study was found to have a significant effect on other-race face recognition. Overall, these findings from the present study lend support for the Categorisation-Individuation model that 


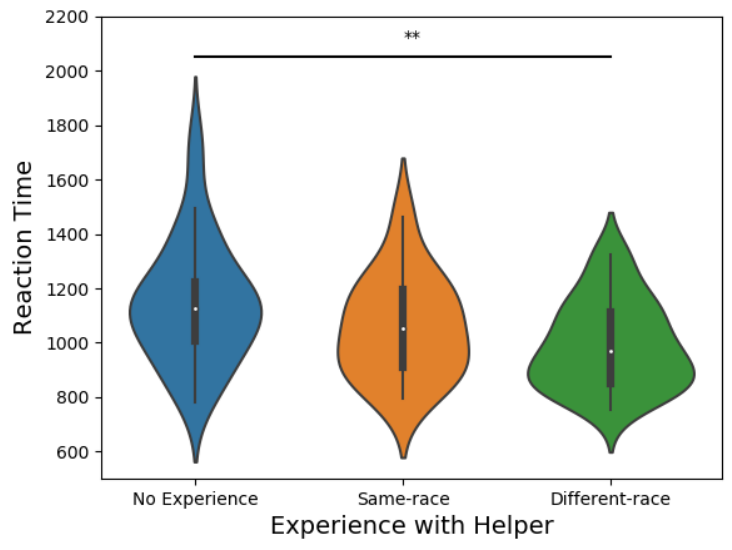

Figure 2. Reaction times of individuals by type of caregiving experience

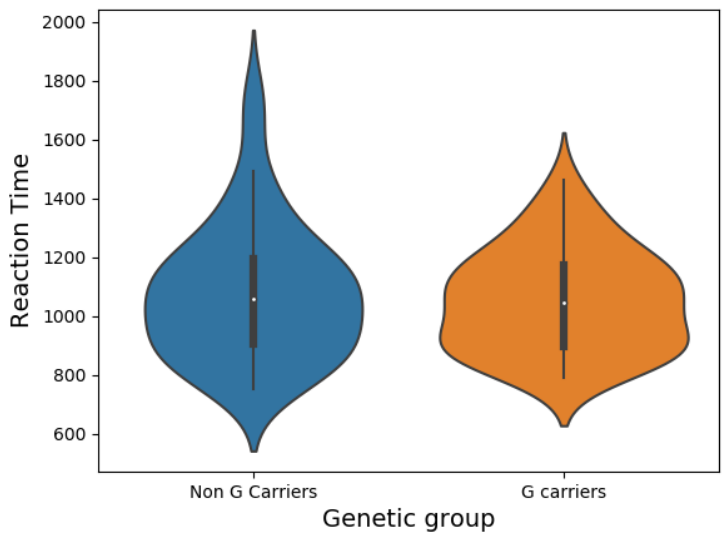

Figure 3. Reaction times of individuals by rs53576 genotype
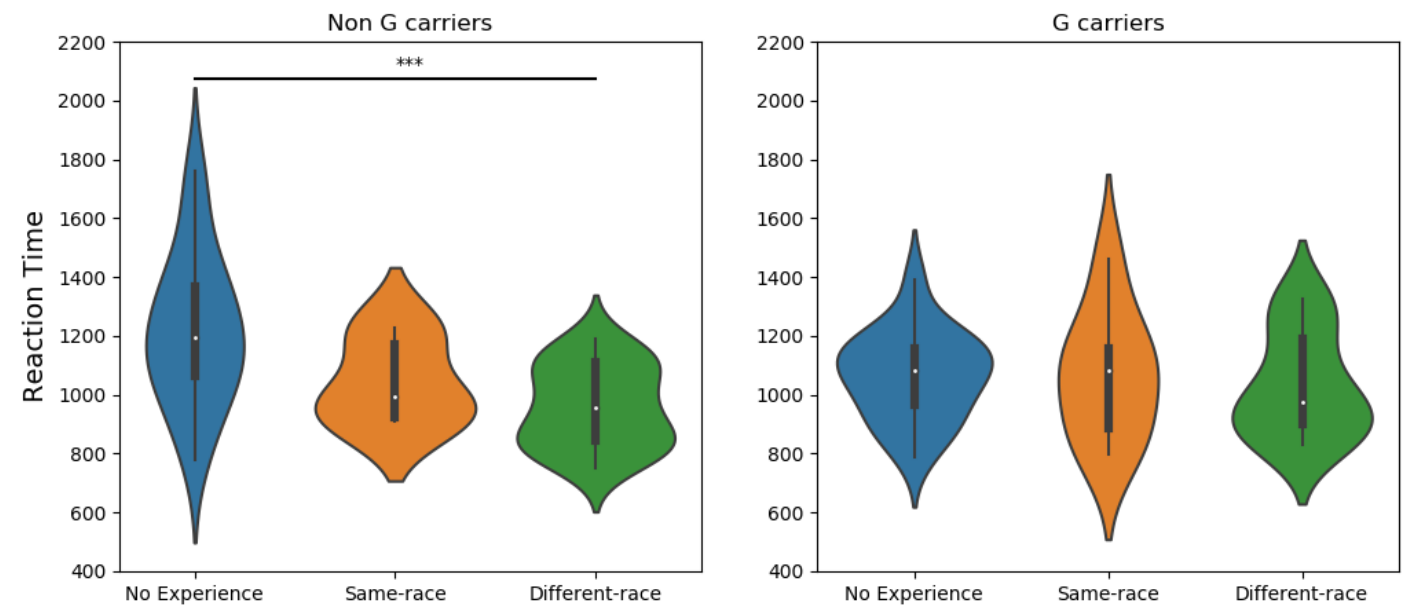

Figure 4. Reaction times by type of caregiving experience grouped by rs53576 genotype 
individuation experience of an individual contributes to the efficiency of race categorization of faces.

Although there was no significant effect of the rs53576 OXTr genotype alone on other-race face recognition, a significant interaction effect of the rs53576 OXTr genotype with the early caregiving experience was found, which supports Hypothesis 2. The gene-environment interaction was such that having an other-race caregiver improved other-race face recognition compared to individuals without caregiving experience, where this was only observed in non-G carriers of rs53576 but not in $\mathrm{G}$ carriers of rs53576. These findings suggest that the environment - early caregiving experience - may be able to compensate for the profile of less adaptive social traits and characteristics that have previously been associated with non-G carriers of rs53576. Overall, these findings also strongly suggest the importance of applying a genetics perspective to examining other-race face recognition given the significant gene-environment interaction discovered in the present study ${ }^{41}$.

The findings of the present study indicate the role of early caregiving experience on other-race face recognition. Given that early caregiving experience appears to improve other-race face recognition, it supports previous findings that training has been shown to significantly improve other-race face recognition ${ }^{6}$ and that training children can reduce implicit racial bias $^{12}$

However, there are a number of limitations to the study. Firstly, early caregiving experience investigated in this study was measured as the presence or absence of an other or same race caregiver. A number of other factors such as time spent with the caregiver and relationship quality with the caregiver could possibly influence the exposure that individuals would have. Hence, future studies can investigate these factors to better understand how these factors may moderate the effect of early caregiving experience on other-race face recognition. Secondly, early caregiving experience was measured retrospectively by asking adult participants to recall their childhood experiences. Future studies can seek to further strengthen current findings by employing a longitudinal design examining other-race face recognition in children as they develop. These studies will contribute to a more accurate depiction of the duration and timing of exposure to other-race caregivers that must elapse for significant differences in recognition accuracy with individuals without caregiving experience to arise.

\section{Methods}

\section{Participants}

Participants $(\mathrm{n}=89$, mean age $=21.85, \mathrm{SD}=1.70$ females $=$ $65)$ were undergraduates compensated with course credit. All participants were ethnic Chinese. The study was approved by the Psychology Programme Ethics Committee at the Nanyang Technological University and the research conducted in this study was performed in accordance with the guidelines set forth by this ethics committee (IRB Number: IRB-2015-08020-01). Written informed consent was obtained from participants prior to the commencement of the study. All data are available at this URL: https://doi.org/10.21979/N9/IWGQ1M

\section{Procedure}

Participants were assessed individually where they were asked to complete a demographics questionnaire at the beginning of the study to obtain information regarding their early caregiving experiences. Participants were asked for the following details: (i) age at the time of the caregiving experience, (ii) duration of the caregiving experience and (iii) nationality of their caregiver. 53 participants had caregiving experience, with the beginning of receiving caregiving ranging from at birth to 10 years old (mean $=3$ years old). The duration of caregiving received ranged from 1 to 17.5 years (mean $=7$ years). Table 1 shows the nationalities of the caregivers. Upon completion of the questionnaire, participants completed a visual categorization task to measure their performance in categorizing faces by race. The task was conducted using a Microsoft Surface Pro tablet with a touch screen, using E-prime 2.0 Professional (Psychology Software Tools, Sharpsburg, PA). Participants were instructed to place their hands at the side of the tablet to standardize the time needed to move their thumb to the response keys. Participants were also reminded to read the instructions carefully and perform the task as fast and as accurately as possible.

\section{Experimental task Face stimuli}

To assess participants' categorizing ability in identifying the race of face stimuli, 4 unique Chinese faces and 4 unique Javanese faces were used as the face stimuli. Indonesian Javanese faces were chosen as a representation of other-race caregivers in Singapore as Indonesians made up the majority of foreign domestic workers in Singapore ${ }^{42}$. All faces, displayed with neutral expressions, were in colour and taken from a frontal view presented on a white background. The face images were standardized at 480 pixels $(17 \mathrm{~cm})$ wide and 600 pixels $(21 \mathrm{~cm})$ high with a resolution of 72 pixels per inch and were processed to be the same elliptical shape and size, with eyes and nose centrally aligned.

\section{Trials}

The visual categorization task consisted of eight test trials where either a Chinese or Javanese face stimulus will be presented in the middle of the display screen with two race labels. These race labels were programmed at the bottom-left or bottom-right corners of the screen as response keys. A new face was presented following the participant's response. The faces were presented in a randomized order. To control for and minimize the effect of side preference, the position of race labels was counterbalanced such that the race labels alternated between left and right positions for each race. For instance, half of the Chinese face stimuli were programmed 


\begin{tabular}{|l|l|l|}
\hline Number of Caregivers & Nationality & Frequency \\
\hline \multirow{4}{*}{ Single } & $\begin{array}{l}\text { Singaporean } \\
\text { (same-race) }\end{array}$ & 14 \\
\cline { 2 - 3 } & Indonesian & 14 \\
\cline { 2 - 3 } & Filipino & 10 \\
\hline \multirow{3}{*}{ Multiple } & $\begin{array}{l}\text { Indonesian } \\
\text { and Filipino }\end{array}$ & 8 \\
\cline { 2 - 3 } & Others & 7 \\
\hline
\end{tabular}

Table 1. Distribution of participants' early caregivers.

\begin{tabular}{|l|l|l|l|}
\hline & Caregiving & $\mathrm{N}(\%$ of genetic group) & RT: Mean (SD) \\
\hline \multirow{3}{*}{ Non G carriers $(\mathrm{N}=46)$} & No Experience & $19(41.3)$ & $1217.33(254.76)$ \\
\cline { 2 - 4 } & Ingroup & $6(13.0)$ & $1043.21(145.96)$ \\
\cline { 2 - 4 } & Outgroup & $21(45.7)$ & $958.72(138.07)$ \\
\hline \multirow{3}{*}{ G carriers $(\mathrm{N}=43)$} & No Experience & $17(39.5)$ & $1065.05(150.23)$ \\
\cline { 2 - 4 } & Ingroup & $8(18.6)$ & $1067.42(217.94)$ \\
\cline { 2 - 4 } & Outgroup & $18(41.9)$ & $1032.52(177.96)$ \\
\hline
\end{tabular}

Table 2. Distribution of the samples in the two genetic groups and types of early caregiving experience.

with Javanese race label on the left and Chinese race label on the right, and vice versa. The same was programmed for Javanese face stimuli. Participants were not provided with feedback indicating whether their response was correct during the test trials.

\section{OXTr genotyping}

Buccal mucosa samples were collected from each participant and genotyped by a laboratory. DNA extraction was performed for each participant using the Oragene DNA purifying reagent and concentrations were assessed using spectroscopy (NanoDrop Technologies, USA). Polymerase Chain Reaction (PCR) was conducted to amplify the target OXTr gene region rs53576 using $1.5 \mathrm{ll}$ of genomic DNA from the test sample, PCR buffer, $1 \mathrm{mM}$ each of the forward and reverse primers, 10 $\mathrm{mM}$ deoxyribonucleotides, KapaTaq polymerase, and $50 \mathrm{mM}$ $\mathrm{MgCl} 2$. The forward and reverse primers used were 5-GCC CAC CAT GCT CTC CAC ATC-3 and 5-GCT GGA CTC AGG AGG AAT AGG GAC-3. The PCR process involved (i) 15 minutes of denaturation at 95 degrees Celsius, (ii) 35 cycles at 94 degrees Celsius (30 seconds), 60 degrees Celsius (60 seconds), 72 degree Celsius (60 seconds) and (iii) 10 minutes of protraction at 72 degrees Celsius. Participants were classified into (i) $G$ carriers- those with at least one $G$ allele (G/G homozygotes or $\mathrm{A} / \mathrm{G}$ )) or (ii) non-G carriers. The averaged distribution of the different genotypes in the Asiatic population is $45-65 \%$ for non-G carriers (i.e. A/A homozygotes) and $35-55 \%$ for G-carriers. The distribution in our sample was $51.6 \%$ non-G carriers and $48.4 \% \mathrm{G}$ carriers. The distribution of the samples between the different types of experiences with caregiver is comparable in the two genetic groups: $\mathrm{X} 2(2, \mathrm{~N}=$ $89)=0.527, \mathrm{p}=.768($ see Table 2$)$.

\section{Data availability statement}

The datasets generated during and/or analysed during the current study are available at https://doi.org/10.21979/N9/IWGQ1M.

\section{References}

1. Goren, C. C., Sarty, M. \& Wu, P. Y. Visual following and pattern discrimination of face-like stimuli by newborn infants. Pediatrics 56, 544-549 (1975).

2. Morton, J. \& Johnson, M. H. Conspec and conlern: a two-process theory of infant face recognition. Psychol. review 98, 164 (1991).

3. Pascalis, O. et al. On the links among face processing, language processing, and narrowing during development. Child development perspectives 8, 65-70 (2014).

4. Meissner, C. A. \& Brigham, J. C. Thirty years of investigating the own-race bias in memory for faces: a meta-analytic review. Psychol. Public Policy, Law 7, 3 (2001).

5. Dwyer, J., Neufeld, P. \& Scheck, B. Actual Innocence: When Justice Goes Wrong and how to Make it Right. A Signet book (New American Library, 2001).

6. Setoh, P. et al. Racial categorization predicts implicit racial bias in preschool children. Child development $\mathbf{9 0}$, 162-179 (2019).

7. Qian, M. K. et al. Perceptual individuation training (but not mere exposure) reduces implicit racial bias in preschool children. Dev. psychology 53, 845 (2017).

8. Nelson, C. A. The development and neural bases of face recognition. Infant Child Dev. An Int. J. Res. Pract. 10, 3-18 (2001). 
9. Sporer, S. L. Recognizing faces of other ethnic groups: An integration of theories. Psychol. Public Policy, Law 7, 36 (2001).

10. Dunham, Y., Chen, E. E. \& Banaji, M. R. Two signatures of implicit intergroup attitudes: Developmental invariance and early enculturation. Psychol. science 24, 860-868 (2013).

11. Rutland, A., Cameron, L., Milne, A. \& McGeorge, P. Social norms and self-presentation: Children's implicit and explicit intergroup attitudes. Child development 76, 451-466 (2005).

12. Xiao, W. S. et al. Individuation training with other-race faces reduces preschoolers' implicit racial bias: A link between perceptual and social representation of faces in children. Dev. Sci. 18, 655-663 (2015).

13. Anzures, G., Quinn, P. C., Pascalis, O., Slater, A. M. \& Lee, K. Categorization, categorical perception, and asymmetry in infants' representation of face race. Dev. science 13, 553-564 (2010).

14. Quinn, P. C., Lee, K., Pascalis, O. \& Tanaka, J. W. Narrowing in categorical responding to other-race face classes by infants. Dev. Sci. 19, 362-371 (2016).

15. Anzures, G. et al. Brief daily exposures to asian females reverses perceptual narrowing for asian faces in caucasian infants. J. experimental child psychology 112, 484-495 (2012).

16. Heron-Delaney, M. et al. Perceptual training prevents the emergence of the other race effect during infancy. PloS one 6 (2011).

17. Rodin, M. J. Who is memorable to whom: A study of cognitive disregard. Soc. Cogn. 5, 144-165 (1987).

18. Hugenberg, K., Young, S. G., Bernstein, M. J. \& Sacco, D. F. The categorization-individuation model: an integrative account of the other-race recognition deficit. Psychol. review 117, 1168 (2010).

19. Lee, K., Quinn, P. C. \& Pascalis, O. Face race processing and racial bias in early development: A perceptual-social linkage. Curr. Dir. Psychol. Sci. 26, 256-262 (2017).

20. Lee, K., Anzures, G., Quinn, P. C., Pascalis, O. \& Slater, A. Development of face processing expertise. The Oxf. handbook face perception 753-778 (2011).

21. Wan, L., Crookes, K., Reynolds, K. J., Irons, J. L. \& McKone, E. A cultural setting where the other-race effect on face recognition has no social-motivational component and derives entirely from lifetime perceptual experience. Cognition 144, 91-115 (2015).

22. Hancock, K. J. \& Rhodes, G. Contact, configural coding and the other-race effect in face recognition. $B r . J$. Psychol. 99, 45-56 (2008).

23. Zhao, M., Hayward, W. G. \& Bülthoff, I. Holistic processing, contact, and the other-race effect in face recognition. Vis. Res. 105, 61-69 (2014).
24. Michel, C., Caldara, R. \& Rossion, B. Same-race faces are perceived more holistically than other-race faces. Vis. Cogn. 14, 55-73 (2006).

25. Tullis, J. G., Benjamin, A. S. \& Liu, X. Self-pacing study of faces of different races: Metacognitive control over study does not eliminate the cross-race recognition effect. Mem. \& cognition 42, 863-875 (2014).

26. McKone, E. et al. A critical period for faces: Other-race face recognition is improved by childhood but not adult social contact. Sci. reports 9, 1-13 (2019).

27. Singapore Department of Statistics. General household survey (2015).

28. Singapore Department of Statistics. Employed Female Residents Aged 15 Years And Over By Occupation And Age Group, (June), Annual (2017).

29. Kim, J. L. S. \& Ling, C. S. Work-family conflict of women entrepreneurs in singapore. Women Manag. review (2001).

30. Wattis, L., Standing, K. \& Yerkes, M. A. Mothers and work-life balance: Exploring the contradictions and complexities involved in work-family negotiation. Соттиnity, Work. \& Fam. 16, 1-19 (2013).

31. Donaldson, Z. R. \& Young, L. J. Oxytocin, vasopressin, and the neurogenetics of sociality. Science 322, 900-904 (2008).

32. Meyer-Lindenberg, A., Domes, G., Kirsch, P. \& Heinrichs, M. Oxytocin and vasopressin in the human brain: social neuropeptides for translational medicine. Nat. Rev. Neurosci. 12, 524-538 (2011).

33. Ebstein, R. P., Israel, S., Chew, S. H., Zhong, S. \& Knafo, A. Genetics of human social behavior. Neuron 65, 831844 (2010).

34. Gillath, O., Shaver, P. R., Baek, J.-M. \& Chun, D. S. Genetic correlates of adult attachment style. Pers. Soc. Psychol. Bull. 34, 1396-1405 (2008).

35. Rodrigues, S. M., Saslow, L. R., Garcia, N., John, O. P. $\&$ Keltner, D. Oxytocin receptor genetic variation relates to empathy and stress reactivity in humans. Proc. Natl. Acad. Sci. 106, 21437-21441 (2009).

36. Tost, H. et al. A common allele in the oxytocin receptor gene (oxtr) impacts prosocial temperament and human hypothalamic-limbic structure and function. Proc. Natl. Acad. Sci. 107, 13936-13941 (2010).

37. Kogan, A. et al. Thin-slicing study of the oxytocin receptor (oxtr) gene and the evaluation and expression of the prosocial disposition. Proc. Natl. Acad. Sci. 108, 19189-19192 (2011).

38. Skuse, D. H. et al. Common polymorphism in the oxytocin receptor gene (oxtr) is associated with human social recognition skills. Proc. Natl. Acad. Sci. 111, 1987-1992 (2014). 
39. Verhallen, R. J. et al. The oxytocin receptor gene (oxtr) and face recognition. Psychol. science 28, 47-55 (2017).

40. Cloutier, J. \& Macrae, C. N. Who or what are you?: Facial orientation and person construal. Eur. J. Soc. Psychol. 37, 1298-1309 (2007).

41. Esposito, G., Setoh, P., Shinohara, K. \& Bornstein, M. H. The development of attachment: Integrating genes, brain, behavior, and environment. Behav. Brain Res. 325, 87 - 89, DOI: https://doi.org/10.1016/j.bbr.2017.03.025 (2017). SI: Development of Attachment.

42. Ministry of Manpower, Singapore. Foreign workforce numbers (2017).

\section{Acknowledgements}

This research was supported by NAP SUG 2015 (GE), Singapore Ministry of Education ACR Tier 1 (GE; RG149/16 and RT10/19), Singapore Ministry of Education Social Science Research Thematic Grant (MOE2016-SSRTG-017, PS), and the National Research Foundation Singapore (NRFF2016-03, JNF).

\section{Author contributions statement}

P.S. and G.E. conceptualized the study. P.S. collected the data; M.T. and J.N.F. analyzed the genetic data; A.B., analyzed the data. M.N. wrote the original draft; all the authors reviewed and edited the submitted version of the article. 